\title{
INVESTIGACIÓN
}

Recibido: 16/03/2021 --- Aceptado: 16/03/2021 --- Publicado: 24/05/2021

\section{PERCEPCIÓN DEL NEUROMARKETING POR PARTE DE LOS JÓVENES ESPAÑOLES}

\section{Neuromarketing perception by young spanish people}

Cristina Martín Bermúdez . Universidad Camilo José Cela. España. cristina.martin2@alumno.ucjc.edu

83i Laura Melendo Rodríguez-Carmona. Universidad Camilo José Cela. España. lmelendo@ucjc.edu

\section{Cómo citar el artículo:}

Martín Bermúdez, C., y Rodríguez-Carmona, L. M. (2021). Percepción del neuromarketing por parte de los jóvenes españoles. Vivat Academia. Revista de Comunicación, 154, 143-165. http:// doi.org/10.15178/va.2021.154.e1341

http:// www.vivatacademia.net/index.php/vivat/article/view/1341

\section{RESUMEN}

El presente trabajo versa acerca del estado del neuromarketing en la actualidad y tiene como objetivo revisar de forma exhaustiva la percepción de los jóvenes, centrándose en el análisis del grado de conocimiento y de la aceptación que genera dicha disciplina en este grupo de interés. La realización de esta publicación se basa en una metodología mixta. En cuanto a la metodología cualitativa, se han realizado entrevistas telefónicas a las siguientes organizaciones: Asociación de Marketing de España, Asociación Española de Neuromarketing y Neurocomunicación, Inside Brain y Sociograph. Por otra parte, en lo que a la metodología cuantitativa se refiere, se ha incluido una encuesta al público objetivo. Tras la investigación, podemos resaltar que el neuromarketing es una disciplina aún desconocida por los jóvenes de nuestro país, es decir, los futuros consumidores. Sin embargo, los expertos auguran que este panorama va a cambiar. En cuanto a la participación, nuestro grupo de interés no ha mostrado recelo y tampoco claras vinculaciones hacia la controversia que suscita esta disciplina.

\footnotetext{
${ }^{1}$ Cristina Martín Bermúdez: Doble graduada en Publicidad y Relaciones Públicas y Protocolo y Organización de eventos por la Universidad Camilo José Cela. En el momento de la realización de este trabajo se encontraba cursando la asignatura Trabajo fin de grado, fruto de la cual surgió esta investigación.
} 
Consecuentemente, la valoración en conjunto de todos los parámetros estudiados es favorable.

PALABRAS CLAVE: Neuromarketing - Consumo - Técnicas de investigación Percepción - Interés - Jóvenes - Conocimiento.

\section{ABSTRACT}

This survey aims at reviewing the current situation of neuromarketing and its perception by young people, focusing on the analysis of the degree of knowledge and acceptance that this discipline generates in this interest group. This publication is based on a mixed methodology. On the one hand, regarding the qualitative methodology, telephone interviews have been conducted with the following organizations: Marketing Association of Spain, Spanish Neuromarketing and Neurocommunication Association, Inside Brain and Sociograph. On the other hand, regarding the quantitative methodology is concerned, a survey of the target audience has been included. After the investigation, we can highlight that neuromarketing is a discipline still unknown to young people in our country, that is, future consumers. However, experts predict that this scenario will change. Regarding participation, our interest group has shown no suspicion about the controversy caused by this discipline. Consequently, the overall assessment of all the parameters studied is favorable.

KEYWORDS: Neuromarketing - Consumption - Research techniques - Perception Interest - Youth - Knowledge.

\section{INTRODUCCIÓN}

La complejidad de los sistemas de mercado actuales y, consecuentemente, de los comportamientos de compra de los consumidores ha hecho de la investigación de mercados una herramienta totalmente indispensable en nuestros días.

A lo largo de los años se ha demostrado que los estudios de marketing tradicionales no resultan totalmente efectivos, ya que las respuestas aportadas por los usuarios no son un fiel reflejo de la realidad. Esta cuestión se debe principalmente a que en el 85\% de las ocasiones nuestras acciones proceden de procesos inconscientes (Zaltman, 2003). No es que mintamos de forma intencionada en las entrevistas o en los focus group, sino que, sencillamente, la mente no ha sido capaz de interpretar de forma racional por qué compramos.

En la actualidad existe una gran inquietud por conocer cómo responde el consumidor ante tal cantidad de oferta en cada decisión. Los expertos buscan desentrañar los procesos mentales que se llevan a cabo con cada estímulo publicitario. Para ello, combinan las técnicas tradicionales con nuevas tecnologías propias de las neurociencias. 
Este nuevo campo denominado neuromarketing ha despertado un gran revuelo a nivel social. No sólo las empresas especializadas han mostrado su interés, sino que también lo ha hecho el público.

\subsection{Justificación.}

Las últimas exploraciones en el ámbito de la comunicación y la publicidad se centran en observaciones neurológicas. La investigación de mercados y la revolución tecnológica son dos campos que siempre han generado una gran controversia. Esto se acentúa aún más cuando dichas disciplinas aúnan sus esfuerzos para conocer al detalle cómo actúan los clientes. Se trata de un tema muy novedoso y difícil de concretar. Por ello, resulta necesario investigar cuál es la opinión pública que se está produciendo al respecto.

El estudio se centra en el colectivo joven al ser los futuros participantes de dichos estudios y, además, un grupo con gran atención en la tecnología (ya que han nacido con ella y tienen un apego mucho mayor) y en el consumo de marcas.

\subsection{Pregunta de investigación.}

El presente informe versa acerca de estado del neuromarketing en la actualidad y revisa de forma exhaustiva la percepción ante esta nueva disciplina, centrándose en el análisis del grado de conocimiento y de la aceptación que genera dicha ciencia en este grupo de interés.

De esta manera la pregunta de investigación quedaría formulada de la siguiente manera: ¿Cuál es el grado de conocimiento y el nivel de aceptación del neuromarketing por parte de los jóvenes españoles?

\section{OBJETIVOS}

\subsection{Objetivo principal.}

El objetivo principal reside en analizar la percepción de dicha ciencia por parte de los jóvenes. Es decir, conocer si consideran la exploración de la mente del consumidor como algo positivo o, por el contrario, no poseen una buena impresión acerca de este concepto.

\subsection{Objetivos específicos.}

1. Apreciar cuál es el nivel importancia que tiene para este grupo la investigación de mercados y las conclusiones obtenidas en este tipo de ensayos.

2. Identificar el grado de conocimiento que adquiere esta disciplina en nuestros días, aspecto reseñable al tratarse de una materia incipiente. 
3. Realizar una comparativa acerca de la conformidad con los métodos tradicionales y con la aplicación de las nuevas tecnologías propias de las neurociencias.

4. Examinar la perspectiva que adquieren los jóvenes acerca de la ética de este tipo de investigaciones.

5. Valorar cuál es el criterio de nuestro público acerca del uso actual de estas técnicas en nuestro país y de su regulación.

\section{METODOLOGÍA}

La realización de esta publicación se basa en una metodología mixta, ya que hace uso tanto de metodología cualitativa como cuantitativa para conseguir una visión más global del tema a estudiar.

\subsection{Metodología cualitativa.}

Con el objetivo de conocer de forma extensa el campo del neuromarketing se ha realizado una primera etapa de análisis y documentación acerca del tema en cuestión.

La indagación ayuda a describir los distintos aspectos que rodean esta nueva técnica; tales como el grado de importancia que adquiere en nuestros días y sus aplicaciones en el ámbito de la comunicación de marca. Asimismo, se fundamenta la percepción ética de diversos profesionales de renombre, lo cual ayuda a concretar las cuestiones que se deben tratar en el resto de los procedimientos: las entrevistas y los cuestionarios al público. Esta observación previa posibilita igualmente un conocimiento más profundo sobre las técnicas y sobre el sector, aspectos de importancia para entender de forma global estos trabajos de investigación.

Posteriormente, tras haber recabado y analizado toda la información, se entrevista a las siguientes organizaciones (las preguntas se pueden ver en los anexos 1 y 2):

- Asociación de Marketing de España (Víctor Conde, Director general)

- Asociación Española de Neuromarketing y Neurocomunicación. AENENE (Antonio González Morales, Presidente)

- Inside Brain. Neuroscience Applied Center (Francisco Arribas Aguilar, Socio Fundador y Director General)

- Sociograph. Marketing Science Consulting (Elena Martín Guerra, Cofundadora y directora de operaciones)

Las opiniones de las personas entrevistadas permiten conocer de forma más exhaustiva qué es lo que está ocurriendo actualmente, y cómo reacciona el público ante ello. Y, por otra parte, las aportaciones de las empresas aportan información sobre el día a día de las personas que se someten a estos experimentos, a qué técnicas se exponen, cómo reaccionan ante cada una o hacia dónde se están guiando los nuevos sistemas de estudio de la mente. 
Las comunicaciones se centran en dar respuesta a unas preguntas previamente diseñadas, las cuales se envían por correo a electrónico a las instituciones para su revisión y preparación por parte de los expertos que van a contestar. Estas tienen un carácter abierto para evitar sesgar opiniones y para lograr conocer la máxima información posible. Las entrevistas han sido realizadas telefónicamente, excepto la de la compañía AENENE que por motivos de disponibilidad se realizó vía mail en su totalidad. Las mismas fueron realizadas en el periodo temporal comprendido entre febrero y mayo de 2018.

\subsection{Metodología cuantitativa.}

Por otra parte, se incluye en la investigación una encuesta al público objetivo, jóvenes entre 18 y 25 años. El tamaño de la muestra fue de 300 individuos y se realizó en el mes de mayo de 2018 mediante un cuestionario online autoadministrado. Las preguntas del cuestionario se pueden ver en el anexo 3.

Mediante las preguntas que componen el sondeo se busca conocer la reacción de los participantes ante la investigación de mercados. Con preguntas tales como si conocen las técnicas del neuromarketing o si estarían dispuestos a someterse a este tipo de pruebas analizamos el nivel de conocimiento que se tienen sobre esta ciencia. Por otra parte, se trata de averiguar la percepción mediante preguntas acerca de la ética y la regulación. Finalmente, tras haber presentado al entrevistado de qué trata el concepto a lo largo de la entrevista, se presenta una afirmación positiva sobre el neuromarketing para comprobar si su opinión ha cambiado tras conocer la materia.

Las respuestas por parte de los encuestados se analizarán de forma paralela con la opinión los expertos entrevistados. De esta manera, se revisará de forma conjunta también el punto de vista institucional y empresarial, consiguiendo una visión del prisma global.

\section{DISCUSIÓN}

El objetivo del presente trabajo reside en analizar la percepción de los jóvenes acerca del nuevo campo del neuromarketing. Para conocer de primera mano la visión de este grupo de interés se ha procedido, tal y como se especifica en el apartado de metodología, a la realización y observación de una encuesta y de varias entrevistas al sector.

\subsection{Primera parte. La investigación de mercados}

La primera cuestión introduce el tema general del estudio, la investigación de mercados, buscando hallar el nivel de participación que tiene nuestro público objetivo. Para ello, se le cuestiona si han accedido a un experimento de esta índole en alguna ocasión. 
La gran mayoría de la muestra ha respondido afirmativamente. El 90\% de los jóvenes ha respondido que se ha expuesto ante estas técnicas, frente a un 10,3\% de respuesta negativa. Esto nos lleva a concluir que solo en torno a 1 de cada 10 encuestados no ha participado con anterioridad en una investigación de mercados.

\subsubsection{Interés generado}

En la segunda pregunta se cuestiona si este ámbito resulta de interés o no, bajo su propio razonamiento.

Las respuestas han sido favorables hacia la validez y el interés que suscitan estos análisis, ya que un 9,7\% ha respondido 'mucho' y un 39\% 'bastante'. Esto nos da un total de un $48,7 \%$ de respuestas dentro del rango de afinidad.

Cabe reseñar que una notable cantidad de individuos han respondido de forma neutra, es decir, 1 de cada 3 encuestados no se ha posicionado. La suma de los campos positivos y neutros asciende a un $83,7 \%$ del total.

En cuanto a las opiniones desfavorables, un $13 \%$ opina que son poco interesantes y un $4 \%$ que no son nada interesantes.

Combinando esta cuestión con la número 1, podemos relacionar si las personas si las personas que se han sometido a algún experimento los consideran interesantes o no (y en qué grado) y, por otra parte, si los que no lo consideran relevante han participado igualmente.

Una vez realizado el cruce de los datos, observamos cómo casi la totalidad del grupo que considera la investigación de mercados como muy interesante o bastante interesante se ha expuesto a uno de estudios, un $92,42 \%$.

La opinión neutral también presenta altos resultados en la comparativa con la primera cuestión, ya que un $87 \%$ de ellos han contestado a un estudio en algún momento.

Es importante detallar que un $87 \%$ de las personas que tienen una mala consideración hacia el interés de la investigación de mercados (poco o nada interesante) también se han sometido.

\subsubsection{Nivel de participación en un estudio tradicional}

Tras averiguar la primera opinión acerca del campo en general, la siguiente consulta trata de analizar si en el momento actual los participantes estarían dispuestos a participar en un estudio tradicional. Se especifica que el estudio está basado en técnicas tradicionales (únicamente) para poder comparar posteriormente esta metodología con 
la nueva ciencia que nos concierne, el neuromarketing, y valorar así si existe un criterio diferenciado ante cada una de ellas.

De nuevo, la contestación por parte de los jóvenes fue positiva. Más del $80 \%$ no tendría problema en manifestar sus hábitos de consumo de telefonía móvil. Mientras que un $16 \%$ se mostraría reticente a proporcionar dicha información.

\subsubsection{Eficacia de las respuestas. Mente consciente vs. Mente inconsciente}

Las siguientes preguntas sirven para introducir el tema de la eficiencia y el subconsciente. Específicamente la cuarta pregunta guarda relación con la efectividad de los estudios cualitativos y cuantitativos anteriormente mencionados.

La información obtenida tiene unos valores igualados. Un 56\% del total considera que no, que las respuestas no serán un espejo de las actuaciones de los consumidores, frente a un $44 \%$ que afirma que sí.

A continuación, se presenta el punto número 5, el cual versa acerca del motor de la elección de una compra. Se cuestiona si elegimos una marca o producto por impulso o emoción o, por el contrario, mediante un análisis basado en el razonamiento.

Del mismo modo, los datos se encuentran altamente igualados, con una diferencia de un $1 \%$ entre las dos opciones posibles. Un 51\% asegura que las compras se fundamentan de forma razonada, mientras que un $49 \%$ concibe esta decisión como irracional.

Una vez finalizado el bloque de estudio, cabe la interrelación de las dos cuestiones que se acaban de tratar. De esta manera, puede evidenciarse la existencia de algún tipo de correspondencia entre las respuestas. Por ejemplo, comprobar si los individuos que opinan que las respuestas que aportan en los análisis no son un fiel reflejo de la realidad también creen que las compras se realizan por impulso.

Se ha estudiado la respuesta número 4, basada en la eficacia; subordinando la pregunta número 5, basada en el motor de elección de compra. En esta observación podemos comprobar cómo de las personas que opinan que los estudios sí reflejan la realidad un $51 \%$ cree que depende de la mente inconsciente, mientras que un $49 \%$ legitima que sus decisiones tienen una fundamentación consciente.

Este dato refleja la similitud de las respuestas, ya que las proporciones tienen una variación del 1\%. Lo mismo ocurre con los participantes que concluyen que los resultados no son reales, siendo en este caso la variación de un $2 \%$.

\subsection{Segunda parte. Neuromarketing}


Seguidamente, tendrá lugar el examen de la segunda parte de la encuesta, la parte correspondiente al estudio de la percepción del neuromarketing como una rama específica dentro de la investigación de mercados.

\subsubsection{Grado de conocimiento}

A modo de introducción al tema se genera la siguiente consulta acerca del grado de conocimiento:

Sea cual sea tu respuesta a la anterior pregunta, debes saber que más de un $80 \%$ de las decisiones de compra que tomas se organizan con la parte inconsciente de tu cerebro. Es decir, solo un $20 \%$ de lo que decides lo haces de forma consciente. Es por eso por lo que los expertos en investigación de mercados han desarrollado una nueva ciencia denominada neuromarketing. ¿Conoces esta herramienta o has oído hablar de ella?

Los participantes responden mayoritariamente que no, concretamente el $64 \%$. Por otro parte, hay quienes afirman conocer esta nueva ciencia, o al menos haber oído algo acerca de su existencia, en este caso el dato corresponde al 36\% de los encuestados.

Este segundo grupo, conocedor de la ciencia, se enmarca sobre todo en la franja de edad entre los 21 y los 22 años, con un $31 \%$ y un $21 \%$ respectivamente. La edad contigua, es decir, 23 años, también presenta un margen relativamente alto en comparación con el resto de los datos (17\%).

Estos resultados coinciden con las opiniones de las entrevistas en profundidad. A continuación, se detallan algunas de las respuestas sobre el desconocimiento del concepto:

Los jóvenes, y en general la mayoría de la población, no conocen esta ciencia. No tienen ni idea de su existencia ni de su aplicación. En realidad, existe una gran confusión debido al alto porcentaje de personas que hablan de manera errónea y sin conocimiento del neuromarketing. Poco a poco se van enterando, intentamos dar clases, participar en eventos, etc. Pero aún hay mucho camino por hacer. (Asociación Española de Neuromarketing, comunicación personal, mayo de 2018).

Desde las compañías dedicadas exclusivamente a este tipo de técnicas se arroja también una posible respuesta al rango de la edad de aquellos jóvenes que sí que conocen el neuromarketing:

En general un joven estudiante no tiene ni idea. Los de marketing saben de qué va. Saben que existe la terminología y tienen, posiblemente, un conocimiento (Inside Brain Neurosciences Applied Center, comunicación personal, mayo de 2018).

Los jóvenes, sobre todo los estudiantes de publicidad, de marketing, de investigación de mercados, incluso de comercio; cada vez van estando más en 
contacto con estas nuevas disciplinas (Sociograph, comunicación personal, mayo de 2018).

Al tratase de una materia aún desconocida por la mayoría, tal y como se ha mencionado anteriormente un $64 \%$ de los jóvenes ni si quiera han oído hablar de ello, se examina también en qué medida se están organizando eventos o campañas para dar a conocer el neuromarketing. A los que los expertos han contestado de la siguiente manera:

Desde hace dos años venimos organizando eventos especializados en neuromarketing para dar a conocer esta ciencia y ponerla en valor (Asociación de Marketing de España, comunicación personal, mayo de 2018).

En este momento destinamos la mayoría del tiempo a investigar, a montar programas formativos, etc, para que las personas puedan formarse (AENENE, comunicación personal, mayo de 2016).

En cuanto a colaboración con universidades, hemos hecho muchos proyectos. Sobre todo, con universidades privadas. Con universidades públicas es muy complicado, porque si quieres hacer algo puede pasar un año hasta que se lleva a cabo, mínimo un año, hasta que te aprueban una pequeña colaboración. Si me piden una ayuda, yo la hago. Si me piden una colaboración con la universidad, un acuerdo, es casi imposible. (Inside Brain Neurosciences Applied Center, comunicación personal, mayo de 2018)

En la Universidad de Valladolid tenemos un laboratorio físicamente, para dar acceso a quien quiera investigar, ya sean profesores, alumnos... También tenemos una sede en la Universidad Complutense de Madrid, en la Facultad de Ciencias de Información. Y colaboramos también con todos los alumnos que nos necesitan desde cualquier otra universidad. No hace falta ser alumno para colaborar, pero estamos dentro de las universidades, lo que favorece que todas estas cosas surjan. (Sociograph, comunicación personal, mayo de 2018)

\subsubsection{Nivel de participación en un estudio de neuromarketing}

La séptima pregunta hace referencia a la participación en un estudio de neuromarketing. Se cuestiona al individuo si en ese mismo momento estaría dispuesto a acceder a una prueba específica de este tipo.

Los resultados demuestran que los jóvenes se han decantado por el sí. Basándonos en los resultados, 3 de cada 4 encuestados, un $75 \%$ del total, formaría parte de un estudio de investigación de mercados que llevase consigo la necesidad de contar con técnicas de neuromarketing. Mientras que solo 1 de cada 4, un 25\%, preferiría no hacerlo.

De nuevo las conclusiones que nos reporta la encuesta coinciden con las valoraciones de las instituciones y de las empresas que forman parte de este estudio:

La que más rechazo podría generar sería la Resonancia Magnética, porque hay que meterse dentro. Y es la que más da la impresión de que pueden estar controlando 
tu cerebro. Aunque la mayoría no debería generar intrusividad, por lo que te digo, no estudian nada fuera de lo común, es como un estudio normal. Y no pueden manipular nada, solo leen lo que está ocurriendo. (Asociación de Marketing de España, comunicación personal, mayo de 2018)

Yo solo estudio con electroencefalografía y periféricos, son totalmente inocuas y no intrusivas, he realizado bastantes experimentos con jóvenes y todos muy bien. Las peores, que desde la asociación no las apoyamos y por supuesto no usamos son prácticamente el resto PET, resonancia magnética funcional (sólo aceptable en investigación para conocer mejor el cerebro y no en estudios de mercados ni opinión), magneto-encefalografía. Desde la AENENE sólo apoyamos las técnicas 0\% intrusiva y 100\% inocuas. (Asociación Española de Neuromarketing, comunicación personal, mayo de 2018)

En algunos casos, como ocurre en la empresa entrevistada Inside Brain, ni quisiera se les especifica a los individuos que el estudio en el que van a formar parte consta de tecnología propia del neuromarketing. Desde la corporación nos comentan el proceso: Nosotros llevamos haciendo estudios desde hace cinco o seis años y antes de venir a la encuesta, al estudio, no les decimos nunca que la encuesta es de neuromarketing, ni que se usa cualquier tipo de estas tecnologías. Y realmente al principio se quedan un poco sorprendidos, pero luego lo aceptan con bastante sencillez. Ósea que la evolución ha sido buena, porque a la gente a la que le hacemos las encuestas no tienen nada que ver con el mundo del marketing, no son profesionales del mundo del marketing, son gente normal. (Inside Brain Neurosciences Applied Center, comunicación personal, mayo de 2018)

Por otra parte, ha de tenerse en cuenta que el motivo de rechazo ante esta ciencia no tiene por qué centrarse en el hecho de llevar puesto ciertos aparatos, sino que puede ser basarse en el concepto del en sí (Inside Brain Neurosciences Applied Center, comunicación personal, mayo de 2018). Es decir, que se puedan conocer sus reacciones neuronales emocionales, de atención, de agrado, etc. sin que ellos las tengan que manifestar.

Otro aspecto que podemos tener en cuenta en la interpretación es la edad. La compañía Inside Brain nos muestra una opinión acerca de cómo puede influir este factor en la percepción de esta ciencia: “Cuanto más jóvenes son las personas que participan, mejor se lo toman. Más les interesa y más les agrada. Genera mucha curiosidad. Cuanto más mayor más rechazo genera" (Inside Brain Neurosciences Applied Center, comunicación personal, mayo de 2018).

Por otra parte, la empresa Sociograph ha creado su propia tecnología patentada, una técnica novedosa que puede resultar mucho menos intrusiva que las existentes hasta el momento. Desde la corporación nos detallan algunas especificaciones:

Entre todas nuestras tecnologías, tenemos una que es única y exclusiva, nuestra propia tecnología: Sociograph, que da nombre a nuestra empresa. Es una tecnología patentada por la Universidad de Salamanca, que lo que hace es 
registrar la actividad eléctrica de nuestra piel en grupo. Es la única técnica de neuromarketing que puede registrar reacciones grupales, el resto son individuales. Al ser grupal, nos da objetividad. Al final podemos aislar los comportamientos que son propios del individuo, los subjetivos, con respecto a la reacción que tuviera cualquier otra persona. (Sociograph, comunicación personal, mayo de 2018)

La existencia de esta nueva técnica, mucho más simplificada y menos intrusiva (University of Oxford, s.f.), desconocida hasta el momento, nos hace partícipes del gran desarrollo que se está produciendo en la aplicación del neuromarketing de forma vertiginosa (Lindstrom, 2010; Schaik, 2013).

Resulta de interés combinar la anterior respuesta de participación en un experimento tradicional (pregunta 3) con la participación en un estudio de neuromarketing.

El 79\% de aquellos que afirmaron que no les importaría someterse a un estudio de mercados tradicional tampoco tendría ningún problema en acceder a uno específico de neuromarketing. El 20\% restante no accedería a la segunda prueba.

Cabe destacar que el $51 \%$ de aquellos que no se someterían a un estudio tradicional, sí que lo harían en el caso de que este fuera de neuromarketing. Esto nos reporta un dato muy llamativo, 1 de cada 2 de aquellos que afirman que no contestarían a unas preguntas sí que se expondrían a un escaneo de sus constantes cerebrales.

\subsection{3. Ética y deontología.}

La siguiente cuestión hace referencia a la ética de la aplicación de técnicas de estudio de la mente para los análisis de marketing.

Casi la mitad de los encuestados, un $46 \%$, no se ha posicionado ante dicha consulta. La otra mitad se encuentra firmemente dividida. Un 7\% considera esta ciencia como muy ética y un $21 \%$ como bastante ética, lo cual nos lleva a un total de un $28 \%$ de opiniones favorables. Mientras que un $18 \%$ piensa que tiene es poco ética y un $8 \%$ nada ética, es decir, un $26 \%$ de opiniones desfavorables.

Al ser este punto un tema crucial en la perspectiva de los jóvenes acerca del neuromarketing se ha cuestionado también a los expertos cuál es su opinión al respecto.

Sí, no habrá ningún problema. Si lo conocen, y lo conocen bien. Si se les cuenta de manera correcta, sí. Lo importante es cómo se cuente. Ya que es una técnica más, una investigación de mercados (Asociación de Marketing de España, comunicación personal, mayo de 2018). 
Entiendo que el que esté a favor de los estudios de mercados para hacer productos mejores lo apoyará y el que no, pues estará en contra. Es un estudio de mercado más solo que en vez de preguntarse cuestiones se registran señales (Asociación Española de Neuromarketing, comunicación personal, mayo de 2018).

Si lo conocen como yo te lo estoy contando sí (es un estudio más de mercado y no se ejerce ningún control). Si no lo conocen y creen que manipula o puede manipular pues les asustará (Inside Brain Neurosciences Applied Center, comunicación personal, mayo de 2018).

La idea de que es importante la manera en la que se cuente es recurrente (Braidot, 2009). Para obtener una respuesta positiva se debe especificar que es una investigación de mercados más y que con ella no se ejerce ningún tipo de control (Feenstra \& Pallarés-Domínguez, 2017).

En el caso de que se informe de una manera sesgada, la opinión púbica será negativa.

En relación con tema que se está abordando, la ética del sector, se formula la siguiente idea:

Crees que las empresas especializadas en neuromarketing... ¿buscan su propio beneficio o buscan tu propio beneficio (es decir, ofrecerte exactamente los productos/ servicios que quieres).

En esta ocasión 3 de cada 4 encuestados afirman que las empresas buscan su propio beneficio, es decir, reportes económicos. Por otro lado, 1 de cada 4, cree que las compañías que llevan a cabo este tipo de estudios buscan tu propio beneficio, quieren entenderte y ofrecerte lo que quieres.

De nuevo cabe la comparación de las dos cuestiones referidas a la ética, es decir, a la valoración y opinión acerca de los fines o beneficios. Aquellos individuos que creen que el beneficio reside en la empresa que lleva a cabo el estudio, y que no opinan de forma neutral, consideran esta ciencia sobre todo poco $(23 \%)$ y nada ética $(10 \%)$. Sin embargo, un $16 \%$ de este grupo considera el neuromarketing como bastante ético. Los que, por el contrario, creen que el beneficio reside en los consumidores, opinan en su totalidad de forma positiva. Considerando sobre todo los valores mucho (34\%) y bastante $(16 \%)$.

Una vez estudiada la percepción acerca del concepto del neuromarketing, el nivel de participación que mostrarían los jóvenes y la valoración de su implicación ética se procede a conocer la percepción sobre la actualidad del sector. Para comenzar, se trata el término de forma general y, después, se profundiza sobre el ejemplo de España.

\subsubsection{Grado de implantación y uso de las técnicas}


La primera cuestión de esta tercera parte del estudio hace referencia a la frecuencia con la que se pueden estar dando este tipo de estudios.

La mayor parte de los encuestados cree que sí que se están utilizando estas técnicas. Un $10 \%$ cree que pueden estar produciéndose 'muy frecuentemente', un 16\% 'frecuentemente' y un $27 \%$ 'ocasionalmente'. Esto nos lleva a un total de un $73 \%$, en torno a 3 de cada 4 participantes.

Cabe destacar que solo un $1 \%$ de los encuestados ha opinado que cree que no se está produciendo ningún tipo de análisis con estas técnicas.

\subsubsection{Regulación legal}

La siguiente pregunta enlaza la misma idea. En este caso, hace referencia a la necesidad de una regulación firme. Los resultados arrojan una clara respuesta hacia la opinión favorable o neutra, ya que ambas suman un 97\% del total.

La pregunta número 13 cuestiona dicha regulación se puede estar dando ya en nuestro país o no. Tras conocer la repuesta, vemos que los jóvenes se decantan por su existencia. Un $82 \%$ de los participantes ha contestado que si, es decir, que creen que ya existe una normativa hoy en día y que existen organismos que rigen su cumplimiento.

Estos datos coinciden con la opinión de la Asociación de Neuromarketing de España, organismo que regulan el ejercicio de esta ciencia. Esta última institución nos relata la siguiente información: "En nuestra asociación contamos con un código ético desde hace cuatro años. Validando la ética y deontología de nuestro sector. Este código puede en todo momento en nuestra página web corporativa" (Asociación de Marketing de España, comunicación personal, mayo de 2018).

\subsubsection{Existencia del denominado 'Botón de compra'}

La última pregunta del cuestionario, la número 14, busca conocer la percepción de los jóvenes ante el neuromarketing una vez que han oído hablar de ello en este estudio. A través de este interrogante se busca conocer cuál es su opinión acerca del posible control:

Multitud de estudios confirman que desde las investigaciones de neuromarketing no se puede ejercer ningún tipo de 'control' sobre los consumidores ya que el escaneado del cerebro nos puede mostrar dónde está el botón clave (es decir qué te gusta o qué quieres comprar), pero no puede cambiarlo ni pulsarlo. ¿Crees que esta afirmación es cierta?

En esta ocasión un 58\% de los encuestados creen que la afirmación es cierta, es decir, que no se puede llegar a manipular ningún pensamiento del consumidor. Por otra 
parte, un $42 \%$ cree que sí que se puede ejercer control gracias a el denominado 'botón de compra.

Los expertos entrevistados para el estudio creen firmemente que ese 'botón de compra' o 'botón clave' no existe:

Ese botón hasta donde tengo entendido es un bulo de los tantos que existen en las redes y los libros sobre neuromarketing. Sí que es posible ver una zona cerebral que se activa al enviar la orden de mover la mano para pulsar en los test de compra, pero no es detectable que quieras comprar, sino que vas a mover la mano. (Asociación Española de Neuromarketing, comunicación personal, mayo de 2018)

No hay botón clave, eso no existe. Eso de lo que se hablaba al principio, de la existencia de un botón de compra, eso no existe. Las decisiones de compra están vinculadas, o asociadas, a un montón de decisiones cerebrales conscientes y la mayoría inconscientes, pero no existe ningún punto en concreto. Todo lo que hacemos consiste en mirar, miramos las reacciones cerebrales de un individuo ante un estímulo. Cuando ve algo, huele algo, saborea algo o escucha algo. Solo vemos lo que está ocurriendo, pero no podemos intervenir, ni queremos intervenir. Me refiero a que con la tecnología que existe en la actualidad solo se permite ver qué ocurre en la mente, no se puede cambiar nada. (Inside Brain Neurosciences Applied Center, comunicación personal, mayo de 2018)

\section{CONCLUSIONES}

Al tratase de una materia incipiente, el neuromarketing es un ámbito que necesita especialmente ser estudiado. Prestigiosas universidades a nivel mundial están publicando informes sobre el tema en cuestión. Sin embargo, se desconoce exactamente qué está ocurriendo en nuestro país. Qué nivel de implementación se está produciendo, con qué tecnologías; y, sobre todo, qué grado de conocimiento y aceptación adquiere socialmente. Tras una profunda documentación, se observa que no hay demasiada constancia al respecto y que podría ser muy interesante conocer qué opinión despierta.

Aunque el estudio sea de neuromarketing, y esta filosofía nos presente que es más efectivo estudiar directamente las respuestas neuronales, en el caso que nos concierne se ha decidido hacer un sondeo. Esto se debe a que para hallar la percepción de los jóvenes buscamos preguntarles si conocen o no la ciencia y si se someterían a este tipo de experimentos. Es por ello, que no podemos utilizar los aparatos propios de las neurociencias, ya que les estaríamos preguntando si conocen algo que ya les tenemos que haber explicado previamente para ponerles el equipo. Además de ello, tampoco podríamos saber el nivel de participación y la justificación ética, porque solo podríamos estudiar el escaneo de la mente de aquellos que hubieran accedido. 
Una vez realizado el análisis exhaustivo, se ha conseguido contestar la pregunta de investigación planteada: ¿Cuál es el grado de conocimiento y el nivel de aceptación del neuromarketing por parte de los jóvenes españoles?

El objetivo general se centraba en conocer si consideran la exploración de la mente del consumidor como algo positivo o, por el contrario, no poseen una buena impresión acerca de este concepto. A consecuencia de la indagación, podemos concluir que los resultados arrojados son favorables. A continuación, se puntualizan cada uno de los parámetros:

El primer objetivo específico trataba de apreciar cuál es el nivel importancia que tiene para este grupo la investigación de mercados y las conclusiones obtenidas en este tipo de ensayos. En torno a la mitad de los participantes (un 48,7\%) los consideran bastante o muy interesantes. Lo cual nos lleva a un resultado muy positivo, la mayoría se posiciona en los valores dentro del rango de afinidad. Un 35\% votó neutro, no se encuentra ni acuerdo ni en desacuerdo. Este dato nos muestra que hay también hay parte de la población que simplemente decide no posicionarse. Por el contrario, encontramos solo un 30\% que los consideró poco o nada interesantes. De esta manera, aún no sabemos si conocen el neuromarketing o no, pero ya sabemos que sí que les parecen interesantes los estudios del campo en general.

Para completar, se ha cuestionado también si las personas se habían sometido a algún experimento. Combinando esta cuestión con la pregunta del interés anteriormente comentada, se ha podido observar que como casi la totalidad del grupo que considera los muy o bastante interesante se ha expuesto a uno de estos estudios. Lo mismo ocurre con la opinión neutral, un $87 \%$ se ha sometido.

Sin embargo, lo más reseñable es que aquellos que los consideraban como poco o nada interesantes también se exponen, en el mismo grado. Casi 9 de cada 10 de estos escépticos ante la investigación de mercados, también contesta a las encuestas de satisfacción, entrevistas, o cualquier otro tipo de indagación. La posible razón es que vivimos tan rodeados y acostumbrados que incluso aquellos que no tienen inclinación contestan igualmente sin oponer ningún tipo de problema, se trata de algo normalizado.

El siguiente objetivo (número 2) tenía como fin identificar el grado de conocimiento que adquiere esta disciplina en nuestros días. A modo de introducción al tema, se les preguntó si creían que los estudios tradicionales eran eficaces y si las compras que realizamos son conscientes o inconscientes. En este punto los resultados han sido muy igualados. Y lo mismo ocurrió cuando se les preguntó acerca del motor de la elección de compra. Con ello vemos que realmente no hay un sentir claro hacia estas cuestiones, las opiniones están totalmente divididas.

Tendría sentido que existiera una relación directa entre ambas preguntas, que aquellos que opinan que las respuestas no reflejan la realidad también creyeran que 
las compras se realizan por impulso. No obstante, la combinación de ambos datos nos aporta conclusiones muy diferentes. Aquellos que defienden la inefectividad de los estudios de investigación de mercados creen a partes iguales que el motor de la compra es tanto consciente (52\%) como inconsciente (48\%). Igualmente ocurre con los participantes que concluyen que los resultados sí son reales y representativos. Las personas encuestadas no tienen claro su posicionamiento ante ambos conceptos.

Acto seguido, se cuestionó si conocían el neuromarketing o si habían oído hablar de ello. Como era de esperar, un $64 \%$ no tenía constancia. Se trata de una materia incipiente, que está empezando a tener relevancia y a ser conocida a nivel profesional pero aún queda un largo camino por recorrer hasta que lo conozca la población en general. Esto coincide perfectamente con las opiniones aportadas por los expertos.

Por otra parte, resultaba interesante saber quiénes podían ser aquellos jóvenes que contestasen afirmativamente. Por ello, se incluyó dicha consulta en las entrevistas. Según nos informan desde las empresas especializadas en el ámbito, los jóvenes en general no conocen esta ciencia y aquellos que lo conozcan serán probablemente estudiantes de materias relacionadas con el marketing (Inside Brain Neurosciences Applied Center, comunicación personal, mayo de 2018; Sociograph, comunicación personal, mayo de 2018). Contrastando estas aportaciones con los datos de edad de los participantes, vemos que esta idea podría encajar perfectamente, ya que los conocedores de esta ciencia se encuentran sobre todo entre los 20 y 21 años, edad universitaria.

También se ha podido observar que este grado de conocimiento va a aumentar en los próximos años. Esto se debe a que, tal y como nos han informado desde las instituciones y las corporaciones, se están comenzando a organizar eventos para dar a conocer dicha materia. La mayoría de estos orientados a profesionales y estudiantes, por lo que la tendencia de conocimiento va a seguir residiendo en las personas dedicadas al marketing. Sin embargo, el desarrollo y la implantación de esta ciencia en nuestro país hará que la población también vaya conociéndola gradualmente.

Esto nos lleva al tercer objetivo específico, la realización de una comparativa acerca de la conformidad con las técnicas tradicionales y con la aplicación de las nuevas tecnologías propias de las neurociencias. Punto que resulta sumamente importante en el análisis, ya que es la referencia con el ámbito que ya conocían.

Con el fin de llegar a conclusiones certeras, se ha analizado de forma diferenciada si los individuos estarían dispuestos a participar en un estudio tradicional, en la pregunta número 3 del cuestionario, y, más tarde, si lo harían en un estudio que contase con un escaneo de sus constantes cerebrales. Sorprendentemente, los resultados son muy parecidos. Un $84 \%$ contestaría a las preguntas tradicionales y un $75 \%$ accedería a la prueba de escaneo. De este modo, se resuelve que no se produce reticencia ante el hecho de colocarse ciertos aparatos para la investigación. La inmensa mayoría de la gente lo ve como un estudio más, solo que mecanizado. 
Además de ello, se ha analizado la combinación de ambas preguntas. Casi todos aquellos que afirmaron que no les importaría someterse a un estudio de mercados tradicional tampoco tendría ningún problema en acceder a uno de neuromarketing. Este dato puede resultar llamativo, ya que podría esperarse una participación algo menos elevada. No obstante, el dato más reseñable es que el $51 \%$ de aquellos que no se someterían a un estudio tradicional, sí que lo harían en el caso de que este fuera de neuromarketing.

Estas respuestas tan positivas pueden deberse sobre todo al rango de edad. Los jóvenes hemos nacido con la tecnología, vivimos pegados a ella y no nos genera ningún tipo de intrusismo. Es más, entiendo que despierta gran interés porque resulta muy atrayente. El hecho de que se estudien tu mente y puedas verlo de primera mano les parece algo curioso a lo que no les importaría someterse.

Por otro lado, el siguiente objetivo específico a cumplir (número 4) versa acerca de la perspectiva que adquieren los jóvenes sobre la ética de este tipo de investigaciones. Cuestión ante la que casi la mitad de los participantes no se han posicionado. Esto puede enlazarse con la pregunta del grado de conocimiento, ya que un $64 \%$ no conocía esta técnica antes de someterse a la encuesta, por ello no saben realmente si está a favor o en contra. Deberían tener más información para que existiera dicha valoración.

Por último, el quinto objetivo específico busca valorar cuál es el criterio del público acerca del uso actual de estas técnicas en nuestro país y de regulación de estas. En primer lugar, se ha preguntado a los jóvenes si creen que se están realizando estudios de neuromarketing en la actualidad en España. La gran mayoría ha respondido de forma positiva (en torno a 3 de cada 4 participantes). La opinión restante, cree que estos estudios se pueden estar produciendo 'raramente' (un 26\%). Cabe destacar que solo un $1 \%$ de los encuestados ha opinado que cree que no se está produciendo ningún tipo de análisis con estas técnicas.

Una vez que nos hemos cerciorado de que creen que está ciencia se da en nuestro país, se procede a cuestionar la regulación vigente. Mayoritariamente consideran que es necesario. En cuanto a si se estarán regulando no dichos experimentos en España, también consideran que sí.

Para terminar, se ha preguntado a los encuestados si creen que mediante esta nueva ciencia las empresas pueden ejercer algún tipo de manipulación sobre los consumidores. Cuestión ante la que un 56\% ha afirmado que el escaneo nos indica donde está el botón clave, pero no puede cambiarlo ni pulsarlo. Por otra parte, un $42 \%$ cree que sí que se puede ejercer control gracias a el denominado 'botón de compra'. Es decir, los jóvenes piensan en cierto modo que algo de manipulación sí que puede existir. 
Dicho término asusta. Se está utilizando mucho en nuestros días, pero no es cierto. Mi opinión es similar a la de los entrevistados. No hay botón clave, eso no existe (Inside Brain Neurosciences Applied Center, comunicación personal, mayo de 2018). Ese botón es un bulo de los tantos que existen en las redes y los libros sobre neuromarketing (Asociación Española de Neuromarketing, comunicación personal, mayo de 2018).

A modo de conclusiones generales podemos resaltar que el neuromarketing es una ciencia aún desconocida por los jóvenes de nuestro país, es decir, los futuros consumidores. Sin embargo, los expertos auguran que este panorama va a cambiar. Cada vez se va a ir conociendo más a nivel profesional y se van a llevar a cabo más estudios, lo que normalizará la técnica en los años venideros. En cuanto a la participación, nuestro grupo de interés no ha mostrado duda o recelo y tampoco claras vinculaciones hacia la controversia que suscita esta disciplina. Por lo tanto, su predisposición es positiva. Por ultimo, opinan que se están aplicando estas técnicas en España, pero creen que sí que están ya reguladas. Consecuentemente, la valoración en conjunto de todos los parámetros estudiados es totalmente favorable.

\subsection{Futuras líneas de investigación.}

Tal y como hemos podido constatar a lo largo del presente estudio, el neuromarketing es una ciencia que ha revolucionado completamente su sector. Aún se están desarrollando las técnicas y dándose a conocer, pero los avances son muy rápidos y notorios.

Una posible vía de estudio sería la realización de una comparativa entre los métodos tradicionales y esta disciplina, orientada a los profesionales que se dedican a la investigación de mercado. Es decir, conocer la opinión de estos acerca de si van a desaparecer o no las entrevistas, encuestas y demás herramientas. Otra posible vía de investigación, complementaria a este estudio, sería analizar la percepción ante este mismo paradigma de otro público de edad más avanzada, como por ejemplo los adultos. La comparativa de ambos podrían darnos pautas muy reveladoras en cuanto a la percepción dependiendo de la edad.

Estos son únicamente algunos ejemplos, no obstante, infinidad de estudios tienen cabida en este nuevo campo aún tan desconocido y sumamente innovador.

\section{REFERENCIAS}

Braidot, N. (2009). Neuromarketing. ¿Por qué tus clientes se acuestan con otros si dicen que les gustas tú? Gestión 2000.

Feenstra, R. A., \& Pallarés-Domínguez. (2017). Debates éticos en torno al neuromarketing político: el avance tecnológico y su potencial incidencia en la 
Martín Bermúdez, C., y Rodríguez-Carmona, L. M.

Percepción del neuromarketing por parte de los jóvenes españoles

formación de la opinión pública. Veritas, (36), 9-28. https://dx.doi.org/10.4067/S0718-92732017000100001

Lindstrom, M. (2010). Buyology: verdades y mentiras de por qué compramos. Ediciones Gestión 2000.

Schaik, K. V. (2013). How Neuroscience Contributes to Neuromarketing. [Bachelor Thesis Conference University of Twente]

University of Oxford. (s.f.). Introduction to FMRI. shorturl.at/eDEL5

Zaltman, G. (2003). How Customers Think. Essential Insights into the Mind of the Market. Harvard Business School Press.

\section{AUTORAS:}

\section{Cristina Martín Bermúdez:}

Es una profesional de la comunicación con experiencia en corporativo y digital en compañías como Ogilvy \& Mather, Repsol y Banco Santander. Doble graduada en Publicidad y Relaciones Públicas y Protocolo y Organización de eventos por la Universidad Camilo José Cela. En el momento de la realización de esta investigación se encontraba cursando la asignatura Trabajo fin de grado, fruto de la cual surgió esta investigación. Actualmente es investigadora en los campos de neuromarketing y big data y cursa el máster de Finance E Data en EAE Business School, además de trabajar en el Banco Santander en el área de Change Management.

\section{Laura Melendo Rodríguez-Carmona:}

Profesor acreditado doctor (ANECA) con una amplia actividad docente e investigadora centrada en líneas de investigación: planificación de medios, comunicación publicitaria, narrativa transmedia, nuevas tecnologías e innovación educativa. Importante actividad con capítulos de libros y artículos de revistas, en muchos casos, fruto de las investigaciones competitivas donde ha participado como investigadora. Profusa actividad en congresos, como ponente invitada y organizadora. Orcid ID: orcid.org/0000-0003-1111-1905

Google Scholar:

https://scholar.google.es/citations?user=f8cNxy4AAAAJ\&hl=es\&oi=ao 
Martín Bermúdez, C., y Rodríguez-Carmona, L. M.

Percepción del neuromarketing por parte de los jóvenes españoles

\section{ANEXOS}

8.1. Anexo 1. Entrevista realizada a las siguientes instituciones: Asociación de Marketing de España y Asociación Española de Neuromarketing y Neurocomunicación (AENENE).

1. El término neuromarketing designa una nueva ciencia aún desconocida por gran parte de la población. Desde vuestra asociación, ¿qué opinión tenéis al respecto? ¿creéis que los encargados de marketing de las empresas conocen estas nuevas técnicas?

2. Concretamente la investigación de mi Trabajo Fin de Grado se centra en la 'Percepción del neuromarketing por parte de los jóvenes españoles. ¿Qué opináis acerca de su grado de conocimiento? ¿Creéis que tienen constancia de la existencia de este tipo de técnicas?

3. Desde vuestra asociación, ¿se organizan formaciones o eventos sobre este campo de estudio? ¿alguno de estos está enfocado a que los consumidores conozcan el neuromarketing?

4. Numerosos artículos demuestran la inefectividad de las técnicas de investigación de mercados tradicionales, tales como las entrevistas y los focus group, ya que realmente la mayoría de las decisiones de compra son subconscientes. ¿Las empresas que siguen invirtiendo millones de euros en estos estudios, por qué creéis que lo hacen?

5. Como expertos en neuromarketing, ¿qué técnicas destacaríais como las más usadas por las empresas actualmente?

6. Volviendo al tema específico de mi estudio, la percepción de los jóvenes. ¿Creéis que a los jóvenes les parecerían intrusivas estas técnicas? ¿Cuál sería la que más rechazo le podría causar? ¿y la que menos?

7. ¿Qué tipo de perfiles forman parte de vuestra asociación? ¿Contáis con algún equipo especializado en neuromarketing?

8. Existe una gran controversia acerca de la posible falta de ética en este campo de estudio. Autores como Dooley (2015) defienden que no se ejerce ningún tipo de 'control' sobre los consumidores ya que el escaneado del cerebro nos puede mostrar dónde está el botón clave, pero no puede cambiarlo ni pulsarlo. ¿Cuál es vuestra opinión al respecto?

9. Volviendo a concretar, ¿creéis que a los jóvenes les parecerá ético, por el contrario, serán reacios ante lo desconocido?

10. Por último, el neuromarketing se enmarca como la investigación del futuro, la solución definitiva en las investigaciones de mercado. ¿Es esta afirmación cierta? ¿Continuará creciendo el sector como lo ha venido haciendo hasta ahora?

8.2. Anexo 2. Entrevista realizada a las empresas especializadas: Inside Brain. Neuroscience Applied Center y Sociograph. Marketing Science Consulting.

1. El término neuromarketing designa una nueva ciencia aún desconocida por gran parte de la población. Desde Inside Brain, como empresa especializada en 
el sector, ¿qué opinión tenéis al respecto? ¿creéis que los encargados de marketing de las empresas conocen estas nuevas técnicas?

2. Concretamente la investigación de mi Trabajo Fin de Grado se centra en la ‘Percepción del neuromarketing por parte de los jóvenes españoles'. ¿Qué opináis acerca de su grado de conocimiento? ¿Creéis que tienen constancia de la existencia de este tipo de técnicas?

3. En cuanto a la tecnología utilizada, ¿con qué equipo contáis en Inside Brain? ¿Por qué utilizáis esos aparatos y no otros?

4. Volviendo al tema específico de mi estudio, la percepción de los jóvenes. ¿Creéis que a los jóvenes les parecerían intrusivas estas técnicas? ¿Cuál sería la que más rechazo le podría causar? ¿y la que menos?

5. ¿Con qué personal cuenta vuestra compañía? ¿Qué tipo de perfil especializado se requiere para este tipo de investigaciones?

6. ¿En alguna ocasión habéis realizado algún tipo de trabajo conjunto con los estudiantes? Ya sea una investigación en la que participasen o simplemente el hecho de haber dado una ponencia en la facultad. *En el caso de que la respuesta sea no, ¿tenéis pensado hacerlo en un futuro?

7. Existe una gran controversia acerca de la posible falta de ética en los estudios de neuromarketing. Sin embargo, autores como Dooley (2015) defienden que no se ejerce ningún tipo de 'control' sobre los consumidores ya que el escaneado del cerebro nos puede mostrar dónde está el botón clave, pero no puede cambiarlo ni pulsarlo. ¿Cuál es vuestra opinión al respecto?

8. Volviendo a concretar, ¿creéis que a los jóvenes les parecerá ético este campo o, por el contrario, serán reacios ante lo desconocido? * ¿Cómo reclutáis a esa gente? *Cuándo llegan y se les explica que el estudio se realiza mediante tecnologías propias de las neurociencias, ¿hay gente que no accede?

9. ¿Creéis que una vez que se les haya explicado que el neuromarketing simplemente buscar comprender mejor al consumidor y cumplir de forma más optima sus expectativas (se detalla en el cuestionario de mi TFG) la percepción de los encuestados podría cambiar?

10. Por último, el neuromarketing se enmarca como la investigación del futuro, la solución definitiva en las investigaciones de mercado. ¿Es esta afirmación cierta? ¿Creéis que continuará creciendo el sector como lo ha venido haciendo hasta ahora?

8.3. Anexo 3. Encuesta a jóvenes de entre 18 y 25 años. Muestra: 300 participantes: Percepción del Neuromarketing por parte de los jóvenes españoles

1. ¿Alguna vez te has sometido a un estudio de investigación de mercados? (Por ejemplo haber valorado cómo ha sido la atención del personal en un establecimiento o haber contestado a una encuesta de satisfacción tras recibir un pedido online) o Sí o No

2. ¿Te parecen interesantes estos estudios? o Mucho o Bastante o Neutro o Otro: o Poco o Nada 
3. Si ahora mismo te ofrecen participar en un estudio de investigación de mercados tradicional (una entrevista o un test) por ejemplo sobre qué marca de teléfono usas y por qué, ¿contestarías a las preguntas?

o Si o No

4. ¿Crees que tus respuestas reflejarían perfectamente la realidad?

o Si o No

5. ¿Cómo crees que realizas la mayoría de tus compras?

o Por impulso o emoción o Razonadamente

6. Sea cual sea tu respuesta a la anterior pregunta, debes saber que más de un $80 \%$ de las decisiones de compra que tomas se organizan con la parte inconsciente de tu cerebro. Es decir, solo un $20 \%$ de lo que decides lo haces de forma consciente. Es por eso que los expertos en investigación de mercados han desarrollado una nueva ciencia denominada neuromarketing. ¿Conoces esta herramienta o has oído hablar de ella?

o Si o No

7. Con tecnologías como las que se muestran en la imagen (encefalografía) se estudian las respuestas neuronales de los consumidores ante determinados estímulos publicitarios. Volviendo al ejemplo anterior, si te ofreciesen participar en un estudio sobre la marca de móvil que usas, pero en este caso tuvieras que llevar unos dispositivos electrónicos que analizasen tus constantes cerebrales, ¿accederías a la prueba?

o Si o No

8. ¿Quién crees que te realizaría la prueba? o Un médico, ya que la tecnología es propia de las neurociencias o Un experto en marketing, ya qué quieren conocer mis comportamientos de compra

Ambos o No sabe/No contesta

9. ¿Te parece ético que se estudie la mente del consumidor mediante estas técnicas?

o Mucho o Bastante o Neutro o Poco o Nada

10. Crees que las empresas especializadas en neuromarketing... o Buscan su propio beneficio o Buscan tu propio beneficio, es decir, ofrecerte exactamente los productos/servicios que quieres

11. ¿Con qué frecuencia crees que se pueden estar utilizando este tipo de técnicas actualmente en España? *

o Muyfrecuentemente o Raramente o Frecuentemente o Nunca o Ocasionalmente

12. ¿Ves necesario que se regulen este tipo de estudios?

o Mucho o Poco o Bastante o Nada o Neutro

13. ¿Crees que pueden existir organismos y asociaciones que ya regulen estas técnicas actualmente?

o Sí o No

14. Multitud de estudios confirman que desde las investigaciones de neuromarketing no se puede ejercer ningún tipo de 'control' sobre los 
Martín Bermúdez, C., y Rodríguez-Carmona, L. M.

Percepción del neuromarketing por parte de los jóvenes españoles

consumidores ya que el escaneado del cerebro nos puede mostrar dónde está el botón clave (es decir qué te gusta o qué quieres comprar), pero no puede cambiarlo ni pulsarlo. ¿Crees que esta afirmación es cierta?

o Si o No 